\title{
Research on Design of Remote Medical Monitoring System Based on TCP - IP
}

\author{
ZHANG Linsheng, a \\ ${ }^{1}$ School of Communication Engineering, Chongqing College of electronic engineering, Chongqing, \\ 401331 \\ a56016681@qq.com
}

\section{Keywords: TCP - IP, Remote Medical, Monitoring Platform}

\begin{abstract}
Remote medical monitoring system will be a sound health insurance system in China, enhance the inevitable development trend of medical services; the same time, achieve the optimal allocation of medical resources. Aiming at the current situation of domestic medical care and combining with the characteristics of telemedicine based on TCP-IP communication technology, a remote health monitoring system based on TCP-IP and its corresponding service support platform are designed and implemented to provide remote health monitoring, Remote diagnosis and treatment and remote first aid and other services. The overall architecture of the system is introduced, and the hardware and software design method of the system is discussed emphatically. The C8051E microcontroller as the core, the use of digital micro-smart sensor S11T 71, achieve system monitoring and control terminal temperature and humidity acquisition and control; through Ethernet access, the use of simplified TCP / IP protocol, and with wireless bridge, data remote Transmission, the system without calibration with good environmental adaptability.
\end{abstract}

\section{Theoretical Introduction of Remote Medical Monitoring System}

With the development of wireless communication technology, telemedicine technology has been rapid development, in the community of digital medical and family health care and other fields play an important role. The development of the mobile Internet has also promoted the development of mobile applications, and the application of telemedicine monitoring in mobile Internet has become a hotspot in the development of the times. Through mobile data acquisition technology, patient monitoring can be free from time and geographical constraints, which is conducive to reducing the cost of treatment and improve the quality of care.

China and the developed countries in the world compared to remote medical care research started late, nearly 10 years, Chinese telemedicine supervision before entering the practical application stage. The current remote medical care system is relatively large fixed, is not conducive to use in the family. Health care related to the smart phone application function is single, the detection of human physiological parameters of a single, and the need for a lot of testing equipment support. Telemedicine will become an effective means to make up for lack of medical resources, and mobile popularization will become inevitable ${ }^{[1]}$.

For some need for real-time continuous monitoring of chronic diseases, remote monitoring can remind patients to detect the disease as soon as possible, take timely emergency measures to provide a better guarantee for the safety of users. Second, telemedicine can be carried out in the family or community, both to save manpower and resources and transportation costs, reduce the majority of the people's medical expenses, the patient's family can also monitor the patient through the network real-time situation, played a family guard role. In the emergency situation, the telemedicine monitoring system through the automatic monitoring and alarm mechanism, the timely positioning of the patient location for emergency ambulance services; doctors through the use of mobile information terminal can complete the mobile office, improve the efficiency of diagnosis and treatment. 


\section{Overall Design Architecture}

Based on TCP / IP protocol remote monitoring system, using high precision, self-calibration, digital micro-smart sensor, and achieve the monitoring terminal and Ethernet connection, with wireless network equipment to achieve a number of remote location in different remote monitoring interaction between the terminal and the host computer. The whole system consists of remote monitoring terminal and host computer, both through wireless Ethernet to achieve data exchange. The monitoring terminal is built with $\mathrm{M} \mathrm{CU}$ as the core. Taking into account the system function expansion and high reliability, low power consumption, real-time requirements, the choice of high-speed MCU C8051F02Q the microcontroller using pipeline architecture, on-chip resource-rich, cost-effective, able to meet the design requirements ${ }^{[2]}$. Fig. 1 shows overall structure of the system.

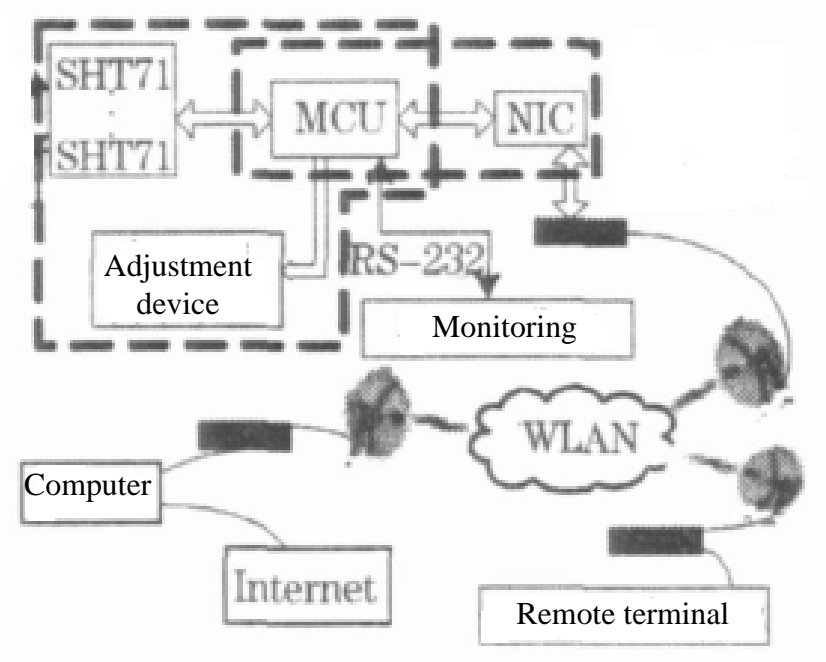

Fig. 1.The overall structure of the system

In order to achieve the monitoring terminal and Ethernet connection, using NI: (network interface chip) to achieve hardware network interface, run by the microcontroller $\mathrm{N}$ IC driver and TCP / P protocol. Since the C8051F020 is an 8-bit microcontroller, select NI: we need to consider whether to support 8-bit operating mode, N IC chip cache size, and the host data exchange form and compatibility with N E 2000. Choose based on the LSA bus 10Mbit / s network interface chip RTL8019A it is cheap, speed and interface are suitable for single-chip system.

In order to avoid the heavy communication line laying and maintenance work, get rid of complex terrain conditions on the system erection, the use of wireless bridge and equipped with parabolic and directional antenna will be remote monitoring terminal access wireless monitoring and control network, establish a connection with the host computer The PC software in Visual C ++ 6. 0 programming environment can be prepared through the wireless link to send control commands, remote configuration of the microcontroller program related parameters, the establishment of the database storage monitoring terminal upload temperature and humidity data and statistical analysis, print statements And temperature and humidity curve. The host computer can also be seamlessly connected with the Internet to achieve a wider range of three monitoring and control ${ }^{[3]}$.

\section{System Implementation}

Remote health monitoring mode can be divided into two kinds of family care and community-based care, guardianship can be divided into continuous or intermittent monitoring two. In this mode, all the guardianship devices in the home are uploaded to the computer or mobile phone via Bluetooth, and then uploaded to the back-end management service platform via the wireless network. After seeing the data, the doctor gives the appropriate health guidance. You can E-MAIL, SMS, etc. to inform customers. In this mode, all of the individual's health data is collected through a multi-function monitoring terminal, and then it is uploaded to the back-end management service 
platform via a wireless network ${ }^{[5]}$. Fig.2 shows medical monitoring system function service.

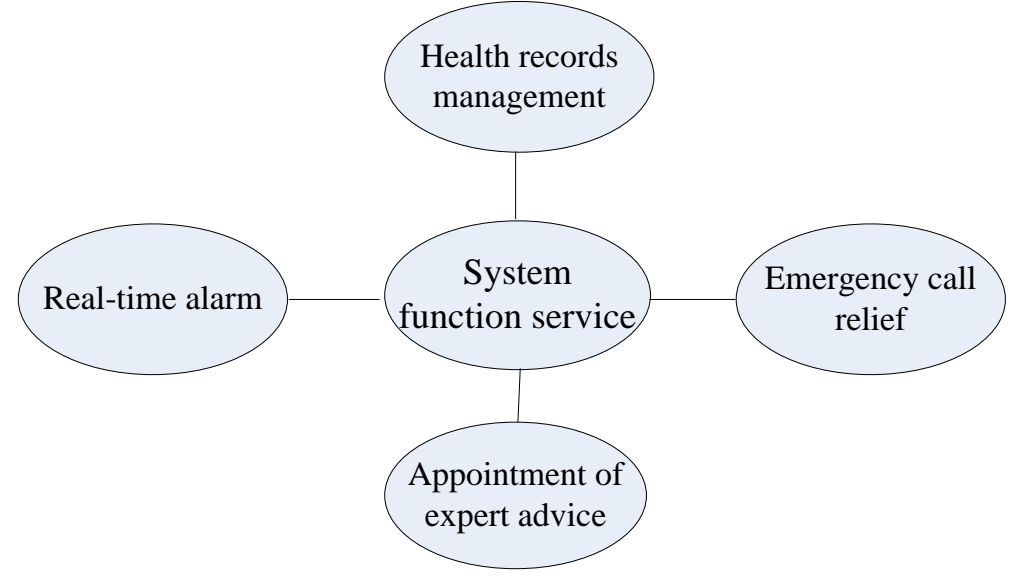

Fig. 2.The medical monitoring system function service

During the monitoring process, when the various types of monitoring physiological parameters are found to be abnormal, the background health management service system can send the health alarm or the prompt information to the guardian's mobile phone or the monitoring terminal through automatic or manual way, or send it to guardian's relatives or other licensors on the phone. Provision of booking expert advisory services can be through the advance appointment of the way, through video and health experts "face to face" exchange, in order to obtain the most timely and most direct health care recommendations. Users can also make an appointment to check their online e-health files online, and by way of SMS to assess the health of the user or give advice.

The system provides emergency call assistance services. When a critical situation occurs, the guardian can dial the telephone of the designated community hospital or health care center by means of a "one-click call" button for emergency call assistance. In addition, the system can also provide home location services. In the monitoring process, the user can not only in the mobile phone or monitoring terminal real-time to see all kinds of monitoring data, but also through the phone, computer Internet for historical data query. The system provides users with lifelong health records management services. In addition, the system also provides 24-hour hotline service.

\section{Remote Monitoring Platform Design}

The remote server receives the monitoring data and the UPS location sent by the patient's smartphone, establishes the database, allows the guardian to access the patient data remotely, and takes the browser / server mode (Browser / Server, B / S) architecture. The remote server analyzes the patient data, gives the initial diagnosis prompt, and divides the red, yellow and green levels into the emergency situation so that the doctor can accord the treatment to the higher risk level. Fig.3 shows physiological parameters sampling module.

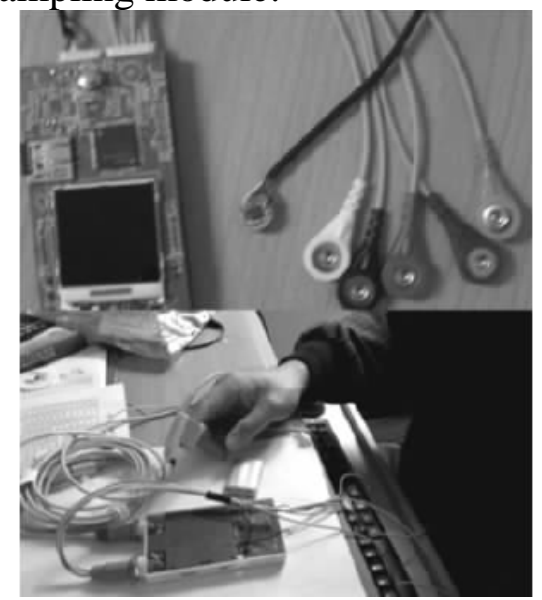

Fig. 3.The physiological parameters sampling module

Health care workers and family members and other caregivers can access the server through the 
Web platform and mobile phone terminal remote access, where the introduction of mobile Android platform client access to facilitate the monitoring staff anytime, anywhere to understand patient information, more closely track and monitor. Patient data in a graphical form to show, to facilitate comparative observation, to the doctor to provide an important basis for clinical diagnosis. The server classifies the remote access users. After the medical staff can visit all the patient information, the disease can be given diagnosis and recommendations. The server can push the patient with a higher critical level of information on the login home page for easy processing.

Family or patient login can view the specified patient information and the doctor's advice. Management personnel can log on to delete historical data and other maintenance operations. The overall design of the remote monitoring system can achieve the sensor of the physiological parameters of the collection, transmission and local smart phone software platform development. The system through the mobile phone as a physiological parameters of the receiving and transit platform, the patient's situation analysis and upload to the Internet, and put forward the central remote server and remote monitoring platform design, through the multi-terminal patients with vital signs remote monitoring, and promote the development of mobile medical.

\section{Conclusions}

Remote health monitoring based on TCP-IP has become an important solution to the current situation of uneven allocation of medical resources. The telemedicine system will improve the medical insurance system of our country and improve the inevitable development trend of medical service. The optimal allocation of medical resources can realize the physical health data of our residents in real time and provide data support and improvement direction for the improvement of the whole people. In this paper, combined with the application requirements and technical characteristics of telemedicine, this paper combines the Internet of Things technology, network and telemedicine information system to provide effective and reliable remote health monitoring, remote first aid and telemedicine services through system solutions and business support platform Of the service; at the same time, saving the patient and medical costs, balanced use of the advantages of medical resources, improve the efficiency of treatment. The system can be applied to remote medical care, maintenance and teaching and home monitoring and other occasions, this remote configuration and monitoring system has a wide range of practical value and social benefits.

\section{References}

[1] Chen Xin. Design of remote medical care system based on smartphone Android platform [D]. Cheng du University of Technology, 2013.

[2] Zhou Kaili, Zhang Wei, Wang Xu of human physiological parameters monitoring system based on Bluetooth wireless and smart phone field .TCP-IP technology, 2015 (6): 18-20.

[3] Zhang Hongbei, Zhao Xiaojun, Li Rui, et al. Design of remote medical monitoring system based on Android. Microcomputer and application, 2015 (19): 64- 66.

[4] Wang Feng, Xuan Bo Kai, Yang Peng. Based on TCP-IP home mobile medical monitoring system design field. Computer measurement and control, 2015, 23 (5): 1586-1588.

[5] Wu Xiaolin, Xu Hongzhi, Huang Yan, et al. Analysis and Design of Medical Monitoring System Based on TCP-IP. Information System Engineering, 2015 (1): 78-79. 